\title{
Computational Prediction of Hot Spots and Binding Site of Inhibitor NSC23766 on Rac1 Binding With Tiam1
}

\author{
Chunwen Zheng ${ }^{1 \dagger}$, Xiaodong $W u^{2,3 \dagger}$, Ruijie Zeng ${ }^{1}$, Lirui $\operatorname{Lin}^{2,3}$, Liyan $X u^{4}$, Enmin $\mathrm{Li}^{2,4 *}$ and \\ Geng Dong ${ }^{2,3 *}$
}

${ }^{1}$ Shantou University Medical College, Shantou, China, ${ }^{2}$ Department of Biochemistry and Molecular Biology, Shantou University Medical College, Shantou, China, ${ }^{3}$ Medical Informatics Research Center, Shantou University Medical College, Shantou, China, ${ }^{4}$ Key Laboratory of Molecular Biology in High Cancer Incidence Coastal Area of Guangdong Higher Education Institutes, Shantou University Medical College, Shantou, China

OPEN ACCESS

Edited by:

Jilai Li,

Jilin University, China

Reviewed by:

Xiang Sheng,

Stockholm University, Sweden

Venkateswarlu Divi,

North Carolina Agricultural and

Technical State University,

United States

*Correspondence:

Enmin $\mathrm{Li}$

nmli@stu.edu.cn

Geng Dong

gdong@stu.edu.cn

${ }^{\dagger}$ These authors contributed equally to this work

Specialty section: This article was submitted to Theoretical and Computational

Chemistry,

a section of the journal

Frontiers in Chemistry

Received: 03 November 2020 Accepted: 29 December 2020 Published: 02 February 2021

Citation:

Zheng $C$, Wu X, Zeng R, Lin L, Xu L, Li E and Dong G (2021) Computational Prediction of Hot Spots and Binding Site of Inhibitor NSC23766 on Rac1 Binding With Tiam1.

Front. Chem. 8:625437.

doi: 10.3389/fchem.2020.625437
Rac1 is a small signaling protein, which belongs to the Rho subfamily of Ras superfamily. It is activated by binding GTP and inactivated by exchanging GDP for GTP. The ability of nucleotide exchange depends on guanine nucleotide exchange factors (GEFs) family proteins. T-lymphoma invasion and metastasis factor 1 (Tiam1) is a member of GEFs. Rac1 participates in multiple signaling pathways and regulates various cellular events by interacting with GEFs. Particularly, it is involved in the development and progression of various kinds of tumors. In this paper, we have studied the detailed interaction between Rac1 and Tiam1. Seven residues on Rac1 are predicted to be important for the interaction with Tiam1, i.e. E31, Y32, D38, N39, Y64, D65 and W56. All these residues are located on the switch 1 and 2 domains which are the interface between Rac1 and Tiam1, except W56. In addition, we analyzed how inhibitor NSC23766 interacts with Rac1. Our docking results show that NSC23766 binds to the same region as Tiam1. Several residues, i.e. F37, D38, N39, W56, Y64, L67, L70 and S71, contribute much to binding free energy. These findings are very useful for the structure-based design of inhibitors toward Rac1.

Keywords: Rac1, Tiam1, NSC23766, molecular docking, MD simulation, binding free energy

\section{INTRODUCTION}

Rac1, a member of Rho family GTPase, is found to involve in the development and progression of various kinds of tumors (Myant et al., 2013; Wang et al., 2015). Rac1 is reported to play both tumorpromoting role and tumor-suppression role in certain kinds of tumors, which indicates that the function of Rac1 remains controversial and is related to a complex network of tumor regulation (Engers et al., 2001; Malliri et al., 2002; Baugher et al., 2005; Chen et al., 2011; Frances et al., 2015). Similar to the other Rho GTPases, Rac1 could causes chemoresistance in various kinds of cancers. It can be turned on and off as a molecular switch and transformed between inactive state with guanosine diphosphate (GDP) and active state with guanosine triphosphate (GTP) (Um et al., 2014; Cardama et al., 2017). When Rac1 is activated, it launches a broad spectrum of downstream pathways and involves in modulating various processes of cancer progression, including cytoskeletal reorganization, migration, invasion and metastasis (Kamai et al., 2010; Myant et al., 2013; Wang et al., 2015). Recently, we found that Racl played an important role of tumor-promoting in the progression of esophageal squamous cell carcinoma (ESCC) and the expression of Racl was 
positively related to cisplatin resistance in ESCC cells (Zeng et al., 2019). Therefore, inhibiting Racl is a solution to inhibit progression of tumors.

Tiam 1 is one of the guanine nucleotide exchange factors (GEFs) those activate small GTPases transforming from GDPbound to GTP-bound state, leading to a tumor-promoting effect (Um et al., 2014; Ruihua et al., 2016; Cardama et al., 2017). In 1994, Habets and his coworkers first identified Tiam1 and found that Tiam1 confers an invasive phenotype to murine T-lymphoma cells (Habets et al., 1994). In the same work, they reported that the function of Tiam 1 is mainly for the activation of Racl by stimulating the exchange of GDP for GTP. During this process, the structure of Tiam1 was suggested to be adjusted for recognition and binding to Racl (Habets et al., 1994; Boissier and Huynh-Do, 2014).

In order to investigate the interaction between Racl and Tiam1, Worthylake et al. determined the crystal structure of $\mathrm{DH}$ and $\mathrm{PH}$ domains of Tiam1 in complex with Rac1 (David et al., 2000). The crystal structure of Tiam1-Racl complex provides novel insights into developing therapeutic strategy to inhibit Rho GTPase activity and the associated downstream pathways (Akbar et al., 2006). By blocking the interactions between Rho GTPases and the specific Dbl family GEFs, the Rho GTPase activity is inhibited and the progression of cancer cells may be arrested.

Based on the crystal structure of Tiam1- Racl complex, the chemical compound NSC23766 was found to targeting to Rac1 by Gao et al. using computational method (Gao et al., 2004). NSC23766 is the first and most widely used Racl inhibitor that binding to Rac1-GEF interface (Levay et al., 2013; Dütting et al., 2015). Owing to the discovery of Racl activation-specific inhibitor, research on cell biological studies of Racl functions and chemotherapeutic targeting at Racl dysregulation is largely promoted (Gao et al., 2004). Recently, our study demonstrated that inhibition of Racl resulted in a significant effect in reversing chemoresistance in ESCC on molecular, cellular and xenograft mice model levels (Zeng et al., 2019). However, the detailed mechanism is unclear.

Despite the crystal structure of Rac1-Tiam1 complex has be solved, the key residues (hot spots) that affect the interaction between Rac1 and Tiam1 is not fully identified. In particular, the mechanism of the binding of NSC23766 has not been systematical studied. In this work, we have studied the detailed interactions between Racl and Tiam1 and between Racl and NSC23766, and predicted the hot spots on Rac1 by theoretical methods. This study provides valuable insights for the cancer treatment based on the inhibition of Racl.

\section{METHODS}

\section{Molecular Docking}

To obtain the complex of Rac1 with NSC23766, we performed the molecular docking calculations. The structure of Racl was used with PDB ID: 5N6O (Ferrandez et al., 2017). Since different scoring functions are used in different docking programs, docking calculations were carried out with three docking programs to make a comparison, i.e. Autodock4, Autodock Vina and HDCOK (Pearson and Lipman, 1988; Berman et al., 2000; Martí-Renom et al., 2000; Larkin et al., 2007; Morris et al., 2009; Trott and Olson, 2010; Remmert et al., 2011; Sievers et al., 2011). The former two programs are implemented in the AMDock software (Valdés-Tresanco et al., 2020). For Autudock 4 and Autodock Vina, the grid boxes were set to the same position as it is in the study by Gao et al. (2004), whereas automatic searching mode was used for HDOCK. All docking calculations were carried out with default parameters. Finally, the best pose from each docking program was used for further studies.

\section{Molecular Dynamics Simulations}

The calculation of Rac1-Tiam1 complex was based on the $2.8 \AA$ crystal structure (PDB ID: 1FOE) (David et al., 2000) and Rac1NSC23766 complex was based on the $2.59 \AA$ crystal structure (PDB ID: 5N6O) (Ferrandez et al., 2017). The Rac1-Tiam1 complex and Rac1-NSC23766 were solvated in a truncated octahedral box extending at least $10 \AA$ on all sides of the complex. The protonation states of all the residues were determined by using PROPKA (Bas et al., 2010), a study of the hydrogen-bond pattern around the His residues, the solvent accessibility, and the possible formation of ionic pairs. All Arg, Lys, Asp, and Glu residues were assumed to be charged. As for histidine, in the Rac1-Tiam1 complex, His1149, 1178, 1216 and 1333 of Tiam 1 and His 104 of Rac1 were assumed be protonated on ND1, and His1214 of Tiam1 was assumed to be protonated on the NE2 atom, besides remaining His residues were modeled as doubly protonated. In the Rac1-NSC23766 complexes, we assumed His104 to be protonated on the ND1 atom and His 103 as doubly protonated.

All the simulations were run using the pmemd module in Amber 18 (Case et al. 2018). By using Langevin dynamics (Wu and Brooks, 2003) we kept the temperate at $300 \mathrm{~K}$, and a collision frequency of $2.0 \mathrm{ps}^{-1}$, the pressure was kept constant at $1 \mathrm{~atm}$ using a weak-coupling isotropic algorithm with a relaxation time of 1 ps (Berendsen et al., 1984). Particle mesh Ewald summation with a fourth-order B spline interpolation and a tolerance of $10^{-5}$ was used to handle the long-range electrostatics (Darden et al., 1993). The SHAKE algorithm (Ryckaert et al., 1977) was used to constrain bonds involving hydrogen atoms so that a 2 fs time step could be used. The cutoff for nonbonded interactions was set to $8 \AA$. The ff14SB force field (Maier et al., 2015) was used for protein and the protein and ligand were solvated in a truncated octahedral box of TIP3P molecules, extending $10 \AA$ from the protein and ligand (Gillan et al., 2016). The AM1-BCC atomic charges (Jakalian et al., 2000; Jakalian et al., 2002) were assigned to each atom of ligand by using the antechamber module (Junmei et al., 2010). the general AMBER force field (GAFF) were used for NSC23776 (Wang et al., 2004).

All simulations were started by a 1000-step minimization, and followed by $20 \mathrm{ps}$ constant-volume equilibration and $20 \mathrm{ps}$ constant-pressure equilibration. The heavy atoms of protein were restrained during the equilibration. Then, 1ns constantpressure equilibration was performed without any restraints. Finally, the 10-ns production simulation was carried out and 


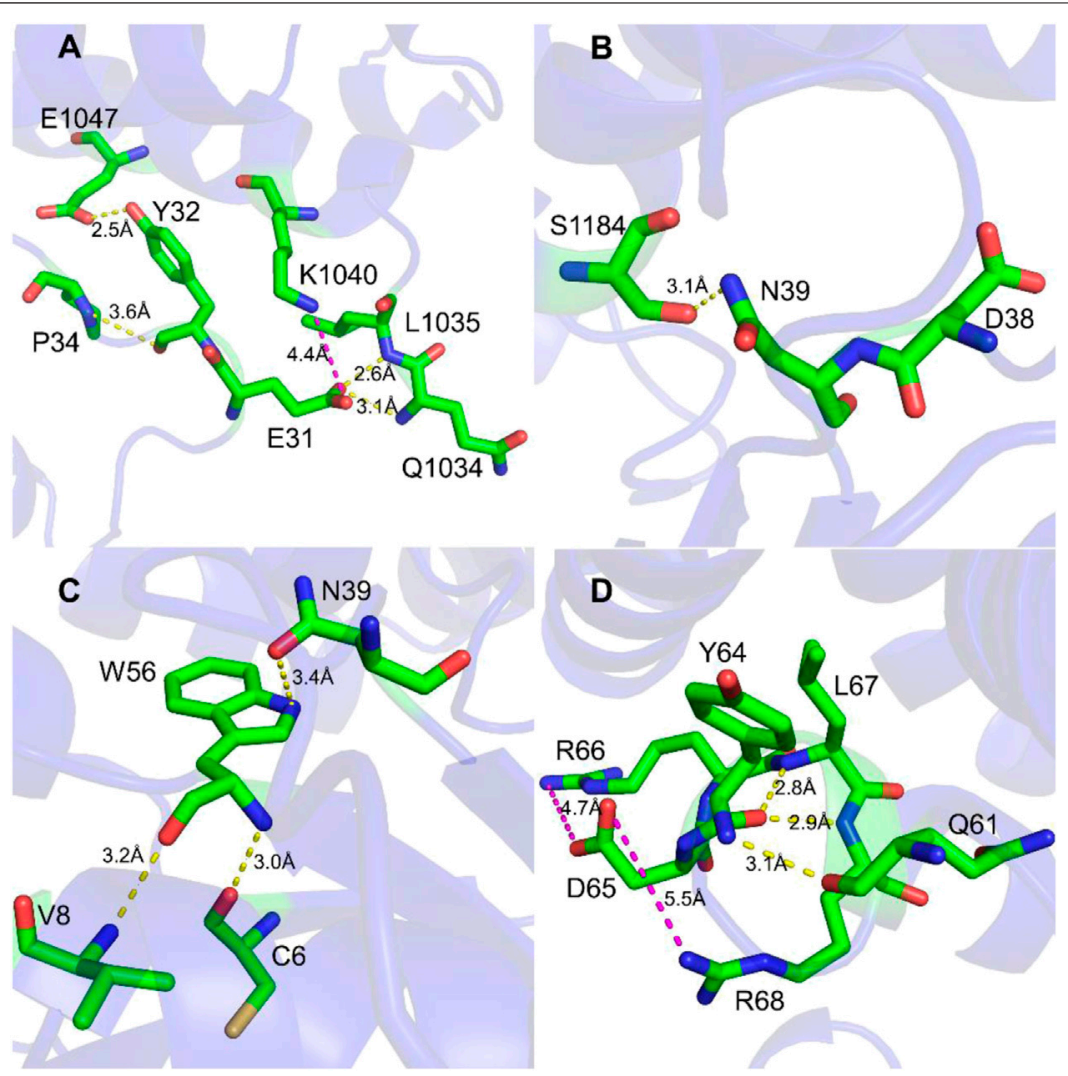

FIGURE 1 | Interaction detail of hot spots in Rac1-Tiam1 structure. The yellow dotted lines represent hydrogen bond, and magenta dotted lines represent saltbridge interaction. (A,B) show interactions formed by residues on switch 1 of Rac1. (A) Interaction around residue E31 $1_{R}$ and $Y_{3} 2_{R}$. (B) Interaction around residue N39 $_{R}$. (C,D) show the intermolecular interactions of switch 2 of Rac1. (C) Interactions around residue W56 $6_{R}$ (D) Interactions around residue Y64 ${ }_{R}$ and D65 $5_{R}$.

coordinates were saved every 2 ps. For the Rac1-Tiam1 system, seven replicates of production calculations were run and three replicates were run for Rac1-NSC23776 system.

Finally we calculated the root mean square deviation (RMSD) and root mean square fluctuation (RMSF) using the cpptrajy module in AMBER 18 (Case et al. 2018).

\section{The Relative Binding Free Energy With Alanine Scanning}

Alanine-scanning calculations were performed for Rac1-Tiam1 system. The alanine-mutant trajectory was obtained from the trajectory of MD simulation for wild-type protein. This strategy of Ala scanning is the simplest and most efficient approach that has been shown to give reasonable results (Kuhn and Kollman, 2000; Huo et al., 2001; Moreira et al., 2007). In this work, we run Ala-scanning calculations for the seven parallel trajectories. Frames from 1,000 to 5,000 were select for alanine scanning calculations. Finally, the average and standard deviation of the binding free energies were calculated.

\section{Absolute Binding Free Energy Calculations With MM/GBSA}

Conventional molecular dynamics simulations were conducted to generate a representative ensemble of structures, and MM/ GBSA method was then used to calculate absolute binding free energies of NSC23776 binding to Rac1 and Tiam1 binding to Racl. In this method, the calculation of $\Delta G_{\text {bind }}$ can be expressed as follow:

$$
\Delta G_{\text {bind }}=G_{\text {complex }}-G_{\text {protein }}-G_{\text {ligand }}
$$

where the binding free energy $\left(\Delta G_{\text {bind }}\right.$ ) is computed as the difference between the free energies of the complex $\left(G_{\text {complex }}\right)$, the protein $\left(G_{\text {protein }}\right)$ and the ligand $\left(G_{\text {ligand }}\right)$.

For all systems, snapshots from the last 8-ns MD trajectory were used. To evaluate the contribution of specific residues to binding free energies, a residue-based free energy decomposition method was employed to calculate the complexes interaction spectrum by using the same snapshots as those used in MM/ GBSA calculations (Gohlke et al., 2003). 


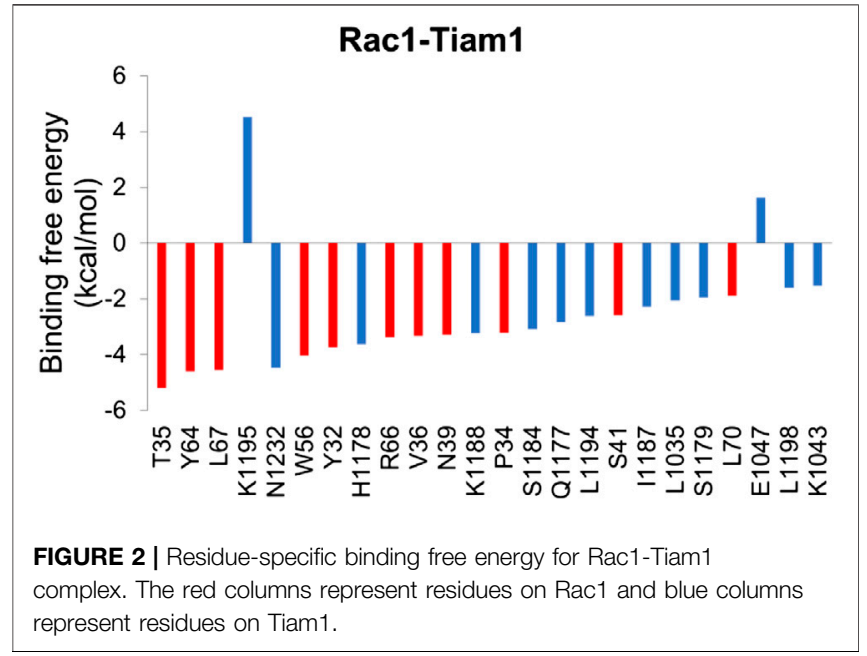

\section{RESULT}

\section{Interaction Analysis Based on MD Simulation}

To study the interaction between Racl and Tiam1, we first performed MD simulation for the Rac1-Tiam1 complex. In the complex, the switch 1 and 2 domains of Racl might be important regions in the interaction with Tiam1, because the two domains locate on the interface of the two proteins. First, we focused on the residues on the switch 1 domain. As is shown in Figure $1 \mathrm{~A}, \mathrm{E} 31_{\mathrm{R}}$ (subscript $\mathrm{R}$ means that residue belongs Rac1) forms hydrogen bond with Q1034 $4_{\mathrm{T}}$ and $\mathrm{L} 1035_{\mathrm{T}}$ (subscript T means that residue belongs Tiam1), and salt bridge with $\mathrm{K} 1040_{\mathrm{T}}$. $\mathrm{Y} 32_{\mathrm{R}}$ forms hydrogen bond with $\mathrm{E} 1047_{\mathrm{T}}$ and $\mathrm{P} 34_{\mathrm{R}}$. This local area contains abundant intermolecular interactions, indicating that the residues on Rac1 in this region are important for the binding with Tiam1, and the mutation of these residues may significantly influence the function of Racl. In addition, the residue N39 $P_{R}$ forms hydrogen bond with $S 1184_{\mathrm{T}}$ (Figure 1B). Next, we analyzed the intermolecular interactions in switch 2 domain. Residues of C6, V8, N39 and W56 on Rac1 directly interact with Tiam $1 . \mathrm{N} 39_{\mathrm{R}}$ and $\mathrm{W} 56_{\mathrm{R}}$ have been validated by previous studies (Gao et al., 2002; Gao et al., 2004). W56 $6_{\mathrm{R}}$ forms three hydrogen bonds with the other three residues, i.e. $\mathrm{N} 39_{\mathrm{R}}, \mathrm{V} 8_{\mathrm{R}}$ and $\mathrm{C} 6_{\mathrm{R}}$. In addition, the intermolecular interactions formed by other residues in switch 2 domain were shown in Figure 1D. Y64 forms hydrogen bonds with $\mathrm{Q} 61_{\mathrm{R}}, \mathrm{L} 67_{\mathrm{R}}$ and $\mathrm{R} 68_{\mathrm{R}}$, and $\mathrm{D} 65_{\mathrm{R}}$ forms salt bridges with R66 $R_{R}$ and $R 68_{R}$. Thus, our results show that residues in switch 1 and 2 domains of Racl play an important role to binding with Tiaml.

\section{Prediction of Hot Spots on Rac1}

In the study by Yi Zheng et al., the binding interaction assay shown that E31, Y32, D38 and N39 have strong effect on Rac1 binding to TrioN (GEF-H1, another Racl binding protein) and binding affinity will be significantly decreased by mutation to alanine (Gao et al., 2002). The mutants Y64A and D65A were also found to decrease the binding affinity of Rac1 interacting with
TABLE 1 | Computational alanine scanning results for Rac1-Tiam1 complex.

\begin{tabular}{llc}
\hline Mutant & \multicolumn{1}{c}{ Domain } & $\boldsymbol{\Delta} \mathbf{\Delta G}^{\mathbf{a}} \mathbf{( k c a l / m o l )}$ \\
\hline E31A & Switch 1 & $-4.9 \pm 1.6$ \\
Y32A & Switch 1 & $-8.6 \pm 0.8$ \\
D38A & Switch 1 & $-6.5 \pm 0.5$ \\
N39A & Switch 1 & $-6.7 \pm 0.5$ \\
Y64A & Switch 2 & $-6.0 \pm 0.5$ \\
D65A & Switch 2 & $-4.3 \pm 1.5$ \\
W56A & Close to switch 2 & $-12.6 \pm 0.6$
\end{tabular}

${ }^{a} \Delta \Delta G=\Delta G$ (wild type) $-\Delta G$ (mutant).

TABLE 2 | Residue-based free energy decomposition for Rac1-Tiam1 complex (kcal/mol).

\begin{tabular}{lccccc} 
Mutant & VDW & Electrostatic & Polar solvation & Nonpolar & Total \\
\hline Y32 & -4.33 & -7.22 & 8.50 & -0.67 & -3.73 \\
N39 & -3.58 & -10.28 & 11.09 & -0.51 & -3.28 \\
W56 & -2.87 & -2.53 & 1.77 & -0.40 & -4.04 \\
Y64 & -5.78 & -2.09 & 4.02 & -0.73 & -4.59 \\
\hline
\end{tabular}

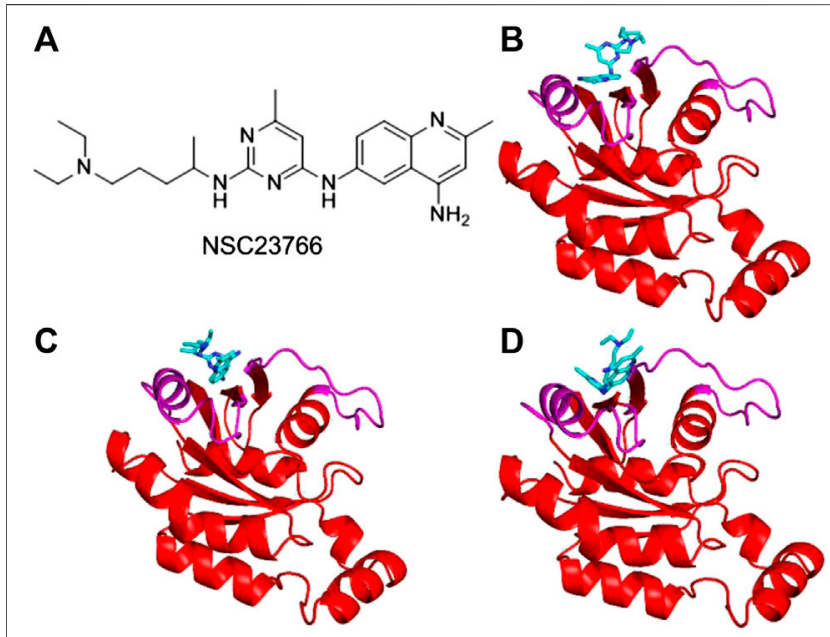

FIGURE 3 | The inhibitor NSC23766 (A) and the docking structures of Rac1 with NSC23766 molecule from Autodock (B), Autodock Vina (C) and HDock (D). The switch 1 and 2 domains of Rac1 were shown in magenta, the NSC23766 molecule was shown as cyan stick model.

TrioN, experimentally (Gao et al., 2002). As we have shown above, E31, Y32, N39, Y64, D65 and W56 have many interactions with the adjacent residues during the MD simulations. We then performed alanine scanning calculations for those seven residues on Rac1, i.e. E31, Y32, D38, N39, W56, Y64 and D65. The alanine scanning results are collected in Table 1. Our calculations show that all mutants decrease the binding affinity of Rac1. D38A and N39A have stronger effect on Rac1-Tiam1 system than E31A in switch 1 domain and Y64A has a larger effect than D65A in switch 2. Our result have a good agreement with experimental data (Gao et al., 2002). In addition, the W56A mutant have the largest effect in our calculations. 


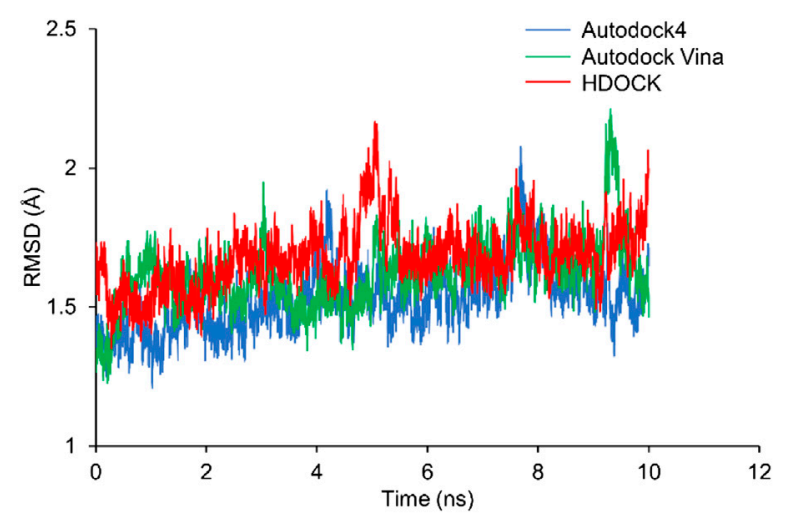

FIGURE 4 | The RMSDs from MD simulations for the three docking structures. The blue, green and red lines are for the structures from Autodock4, Autodock Vina and HDOCK, respectively.

In order to understand the contribution of each residue on Rac1 to the total binding free energy, residue-based free energy decomposition was carried out. In WT Rac1-Tiam1 complex, residues Y32, N39, W56 and Y64 contribute much to the binding free energy, $-3.73,-3.28,-4.04$, and $-4.59 \mathrm{kcal} / \mathrm{mol}$, respectively. The contributions of VDW, electrostatic, polar solvation and nonpolar are shown in Table 2. As we have discussed before that these residues form strong interactions with the residues on Tiam1. In addition, our calculations show that residues T35, L67, R66, V36, P34 and L70 have a large contribution and all of them locate in switch 1 or 2 domains of Rac1(Figure 2). Among these residues, P34, T35 and V36 form hydrogen bond with $\mathrm{E}^{1047_{\mathrm{T}}}$ on Tiam1, the R66 forms hydrogen bond with $\mathrm{N}_{1232_{\mathrm{T}}}$ and salt bridge with E1239 $\mathrm{T}$, the L67 forms hydrogen bond with residues W64 and L70. These interactions might play important roles in Rac1 binding with Tiam1. Therefore, based on previous studies and our calculated results, the switch 1 and 2 domains of Racl are important for the binding with Tiam1.

\section{Molecular Docking}

Inhibitor NSC23766 (Figure 3A) was developed by Zheng and coworkers to specifically block the binding of Tiam1 to Rac1(Gao et al., 2004). However, no complex structure of Rac1-NSC23766 was captured so far. Therefore, we performed molecular docking to obtain the complex of Rac1 with NSC23766. In this work, three docking programs were used, i.e. Autodock4, Autodock Vina and HDock. The best pose of each program was selected for the further studies (Figure 3). The regions shown in magenta in Figure 3 are the switch 1 and 2 domains of Racl. Our docking results show that NSC23766 binds at switch 1 and 2 domains, which are the same regions for Racl interacting with Tiam1. For structure from Autodock, NSC23766 forms hydrogen bond with D57, and NSC23766 located at the hydrophobic pocket produced by residue F37, D38, N39, W56, A59, Y64, L67, R68 and S71. For the structure from Vina, NSC23766 forms hydrogen bond with D57, and NSC23766 located at the hydrophobic pocket formed by residue F37, D38, N39, W56, T58, A59, Y64, L67, R68, L70 and S71. For structure from HDock, NSC23766 forms hydrogen bond with D57, and NSC23766 located at the hydrophobic pocket produced by residue F37, D38, N39, W56, A59, Y64, L67, R68, L70 and S71.

\section{RMSD Analysis}

RMSD shows how much the structure differs from the reference structure. The crystal structure of Rac1 (PDB ID: 5N6O) was used as the reference. All the three docking structures were optimized by $10-n s$ MD simulations, and all simulations were repeated 3 times. RMSD of Rac1 were calculated. Our results show that RMSDs for all the replicates are almost identical (Supplementary Figure S1). Thus, only one of the RMSDs for replicates was selected in Figure 4. It can be seen from Figure 4 that no

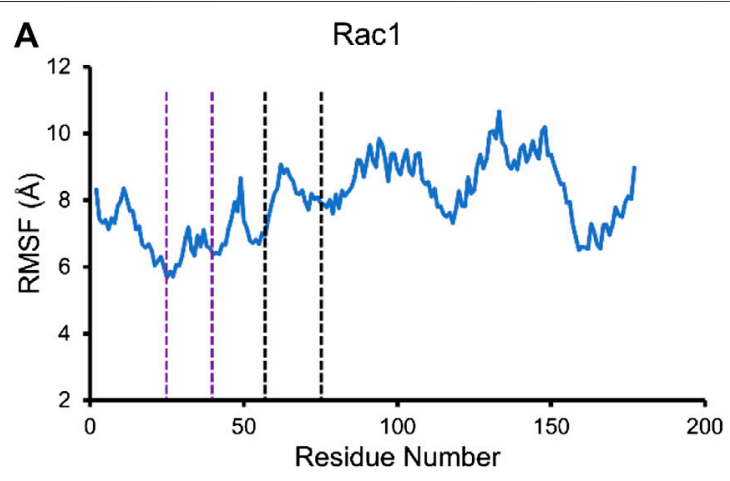

B Rac1-Tiam1
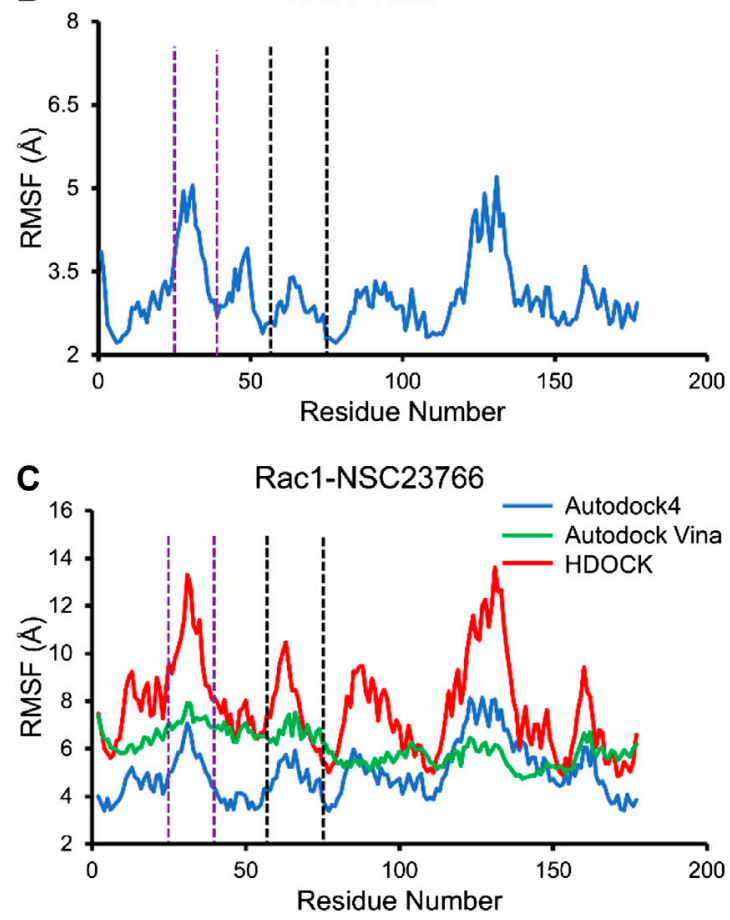

FIGURE 5 | The RMSF for all simulated system. The residues between two purple dotted lines are located at switch 1, and those between two black dotted lines are located at switch 2. (A) RMSF of Rac1 in isolated Rac1. (B) RMSF of Rac1 in Rac1-Tiam1 system. (C) RMSF for three Rac1NSC23766 complex. 


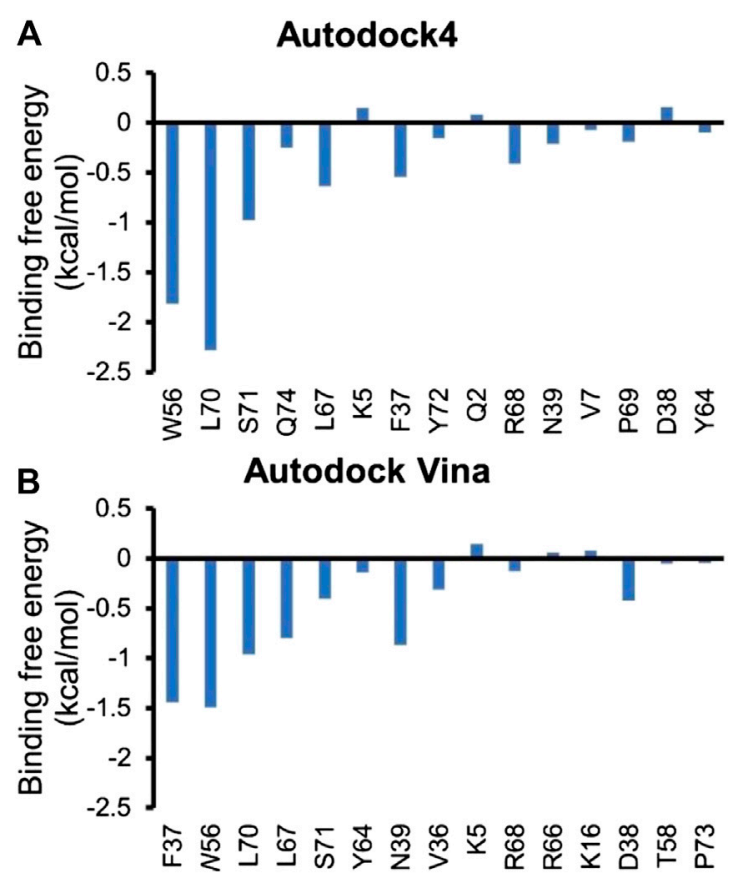

FIGURE 6 | Residue-specific binding free energy for Rac1-NSC23766 complexes.

significant difference is found on Racl among the three simulations for three structures, and all of them are around $1.5 \AA$. Therefore, our results indicate the NSC23766 molecules in the three structures have a similar effect to Rac1.

\section{RMSF Analysis}

Next, we calculated the RMSF of Rac1-Tiam1 complex, Rac1NSC23766 complexes, as well as Rac1 protein. For isolated Rac1, it can be seen from Figure 5A that fluctuations of all residues are above $6 \AA$ and the fluctuations of residues in switch 1 domain (residues 25-39, between purple dotted lines) are lower than those in switch 2 and other regions. When Tiam1 binds to Rac1, (Figure 5B), the fluctuation of the whole system is lower than it in only Rac1 protein. In addition, in Figure 6C, the RMSF of the residues in switch 1 and 2 domains are larger than those residues in other regions, except residues 120-150. In addition, the RMSF of Racl from HDOCK is larger than the other two.

\section{The Prediction of Hot Spots on Rac1 in Rac1-NSC23766 Complex}

In this study, three docking programs were used for prediction of structure of Rac1-NSC23766 complex and the best pose from each software was selected. Our docking results show that NSC23766 molecules in the three docking structures reside in the same area with different poses. Next, we calculated the binding free energy for these structures with MM/GBSA, and the results are collected in Table 3. It can be seen that Autodock4 structure gives a lowest binding free energy, which is 0.3 and $2.4 \mathrm{kcal} / \mathrm{mol}$ lower than Autodock Vina and HDOCK structures,
TABLE 3 | Binding free energy from MM/GBSA for Rac1-Tiam1 complex.
Docking programs

Autodock4

Autodock-Vina

HDOCK
$\Delta \mathrm{G}(\mathrm{kcal} / \mathrm{mol})$

$-16.8$

$-16.5$

$-14.4$ respectively. The highest binding free energy from HDOCK structure might be caused by the large fluctuation (Figure 5C). Thus, the result from HDOCK suggested to be ignored.

Next, residue based binding free energy decompositions were calculated. It can be seen from Figure 6 that the residues those have the large contribution to binding locate in switch 1 and 2 domains. Some of the hot spots in Rac1NSC23766 complex are also important for the interaction between Rac1 and Tiam1, eg W56, L70, etc. Thus, the inhibitor NSC23766 binds to Rac1 and interacts with residues those are important for binding to Tiam 1 to blocks the Tiam1 to interact with Rac1.

\section{DISCUSSION}

With the advances in cancer therapeutics, chemoresistance that leads to impaired treatment efficacy or treatment failure, becomes a great challenge of current clinical practice and scientific research. Individualized evaluation and targeted therapy are popularly pursued. Increasing numbers of studies are carried on to identify the key molecules that modulate chemoresistance and to develop the corresponding drugs to reverse the resistance (Gatenby, 2009; Alfarouk et al., 2015). As a member of Rho family GTPases, Rac1 is widely reported to involve in the development of various malignancies and to modulate chemoresistance or enhance chemosensitivity through a wide spectrum of downstream pathways (Shen et al., 2004; Lee et al., 2007; Zeng et al., 2019; Zhou et al., 2019). Since the activation of Racl is regulated by GEFs, targeting Rac1-GEF complex is taken advantage to develop cancer therapeutics (Gao et al., 2004; Akbar et al., 2006). By using some small molecules/inhibitors of Racl, we can prevent the formation of Rac1-GEF complex to inhibit the activation of Rac1.

Previous experimental study has identified some crucial residues that affect the interaction between Rac1 and Tiam1 (Worthylake et al., 2000; Gao et al., 2002). Our work herein confirms the crucial role of these residues based on molecular docking and MD simulations and predicts hot spots on Racl and the binding sites of NSC23766 molecule. In this work, three programs, i.e. Autodock, Autodock Vina and HDOCK, were used for docking study and the best pose from each software was selected to discuss the computational prediction of hot spots and binding sites of NSC23766 on Rac1 in Racl-Tiam1 interaction. Our results show that the structure from Autodock Vina and Autodock 4 give low binding free energies. The binding pose predicted by HDOCK gives the highest binding free energy and large RMSF. Hence, the poses from Autodock4 and Autodock 
Vina are suggested to be the poses for NSC23766 binding with Racl.

To find hot spots on Rac1, alanine scanning calculations were carried out. Some of these residues were already mentioned as important residues in previous experimental investigations on the interaction between Rac1 and NSC23766. Gao et al., 2002 generated a panel of point mutants at both switch 1 and 2 domains, and found out that D38A and N39A were inactive species for GEF binding activity and GEF responsiveness (Gao et al., 2002). Similarly, Q61L, Y64A and R66A/L67A species lost their binding activity and failed to undergo nucleotide exchange (Gao et al., 2002). Our alanine scanning results (Table 1) agree with the experimental data. We then analyze the detailed interactions and find that all these resides form strong interaction with the residues on Tiam1. In addition, our study predicts that W56 could be a hot spot in both Racl-Tiam1 and Rac1-NSC23766 complex. Based on the crystal structure, we can find that W56 locates in the region near the switch 2 domain and it interacts with N39, C6 and V8 by forming hydrogen bonds.

To obtain binding free energy contribution by each residue that involves in the interaction, we run residue based binding free energy decomposition, which is illustrated in Figure 6. Our results show that D38, N39, Y64 and L67 contribute much to binding free energy, which is consistent with the previous experimental studies (Gao et al., 2002). In addition, our calculations prediction some more residues that largely contribute the binding free energy, i.e. F37, W56, L70 and S71. Although we mainly focus on switch 1 and 2 domains, the functions and conformational changes of the resting regions of Rac1 are also noteworthy. There could be some allosteric sites far from the binding site. Nevertheless, no experimentally approved regions or residues are reported. Our study highlights these residues in the hope that they can gain attention from the scientific community, since they are likely to be key residues influencing Rac1-GEF and Rac1-NSC23766 interactions.

Accumulating evidence shows that residues in switch 1 and 2 domains of Rac1 play a significant role in Rac1-GEF and Rac1NSC23766 interactions. Those residues contribute much to the binding free energy. Mutation of those residues could significantly change the structure and activity of Racl. On the other hand, based on the interactions in this region, we could design new inhibitors to deactivate Racl.

In conclusion, we have predicted the binding site of NSC23766 on Rac1. Our results demonstrate that the inhibitor resides in the region belongs to switch domains 1 and 2. In addition, some residues on Racl are found to be important to interact with Tiam1

\section{REFERENCES}

Akbar, H., Cancelas, J., Williams, D. A., Zheng, J., and Zheng, Y. (2006). Rational design and applications of a Rac GTPase-specific small molecule inhibitor. Methods Enzymol. 406, 554-565. doi:10.1016/S0076-6879(06)06043-5

Alfarouk, K. O., Stock, C. M., Taylor, S., Walsh, M., Muddathir, A. K., Verduzco, D., et al. (2015). Resistance to cancer chemotherapy: failure in drug response from ADME to P-gp. Cancer Cell Int. 15, 71. doi:10.1186/ s12935-015-0221-1 and NSC23766. The detailed interactions of those key residues are studied. This would be very important for the development of nextgeneration drugs which have better effects on blocking Racl interacting with GEFs. Thereby, Rac1 activation by Tiam1 can be inhibited. This would be very important for reducing the influences of Racl on cellular functions of cancer cells to reverse chemoresistance and improve therapeutic efficacy. With the discovery of novel functions of Rac1 in other diseases, Rac1GEF inhibitors are prone to provide more promising treatment options. In addition, predictions of hot spots and binding site are fast and cheap methods for guiding site-specific mutations, and these would provide new insights into the detailed mechanism of inhibitor interaction with target protein.

\section{DATA AVAILABILITY STATEMENT}

The raw data supporting the conclusions of this article will be made available by the authors, without undue reservation.

\section{AUTHOR CONTRIBUTIONS}

All authors listed have made a substantial, direct, and intellectual contribution to the work and approved it for publication.

\section{FUNDING}

This investigation has been supported by grants from This investigation has been supported by the Special Funds for the Cultivation of Guangdong College Students' Scientific and Technological Innovation ('Climbing Program' Special Funds, pdjh2019a0182), the National Undergraduate Training Program for Innovation and Entrepreneurship (201810560037), 'Young Physician Scientist Cultivation' Program of Shantou University Medical College-Li Ka Shing Foundation, 2017-2020 (SMLYPSC-01), and the National Natural Science Foundation of China (21907063).

\section{SUPPLEMENTARY MATERIAL}

The Supplementary Material for this article can be found online at: https://www.frontiersin.org/articles/10.3389/fchem.2020.625437/ full\#supplementary-material.

Bas, D. C., Rogers, D. M., and Jensen, J. H. (2010). Very fast prediction and rationalization of $\mathrm{pKa}$ values for protein-ligand complexes. Proteins 73, 765-783. doi:10.1002/prot.22102

Baugher, P. J., Krishnamoorthy, L., Price, J. E., and Dharmawardhane, S. F. (2005). Racl and Rac3 isoform activation is involved in the invasive and metastatic phenotype of human breast cancer cells. Breast Cancer Res. 7, R965-R974. doi:10.1186/bcr1329

Berendsen, H. J. C., Postma, J. P. M., Van Gunsteren, W. F., Dinola, A., and Haak, J. R. (1984). Molecular dynamics with coupling to an external bath. J. Chem. Phys. 81, 3684-3690. 
Berman, H. M., Westbrook, J., Feng, Z., Gilliland, G., Bhat, T. N., Weissig, H., et al. (2000). The protein data bank. Nucleic Acids Res. 28, 235-242. doi:10.1093/nar/ 28.1.235

Boissier, P., and Huynh-Do, U. (2014). The guanine nucleotide exchange factor Tiam1: a Janus-faced molecule in cellular signaling. Cell. Signal. 26, 483-491. doi:10.1016/j.cellsig.2013.11.034

Cardama, G. A., Gonzalez, N., Maggio, J., Menna, P. L., and Gomez, D. E. (2017). Rho GTPases as therapeutic targets in cancer (Review). Int. J. Oncol. 51, 1025-1034. doi:10.3892/ijo.2017.4093

Case, D. A. I.Y.B.-S., Brozell, S. R., Cerutti, D. S., Cheatham, T. E., Iii, Cruzeiro, V. W. D., et al. (2018). Amber 2018. San Francisco: University of California.

Chen, Q. Y., Xu, L. Q., Jiao, D. M., Yao, Q. H., Wang, Y. Y., Hu, H. Z., et al. (2011). Silencing of Rac1 modifies lung cancer cell migration, invasion and actin cytoskeleton rearrangements and enhances chemosensitivity to antitumor drugs. Int. J. Mol. Med. 28, 769-776. doi:10.3892/ijmm.2011.775

Darden, T., York, D., and Pedersen, L. (1993). Particle mesh Ewald: an N.log(N) method for Ewald sums in large systems. J. Chem. Phys. 98, 10089.

Dütting, S., Heidenreich, J., Cherpokova, D., Amin, E., Zhang, S. C., Ahmadian, M. R., et al. (2015). Critical off-target effects of the widely used Racl inhibitors NSC23766 and EHT1864 in mouse platelets. J. Thromb. Haemostasis 13, 827-838. doi: $10.1111 /$ jth.12861

Engers, R., Springer, E., Michiels, F., Collard, J. G., and Gabbert, H. E. (2001). Rac affects invasion of human renal cell carcinomas by up-regulating tissue inhibitor of metalloproteinases (TIMP)-1 and TIMP-2 expression. J. Biol. Chem. 276, 41889-41897. doi:10.1074/jbc.M105049200

Ferrandez, Y., Zhang, W., Peurois, F., Akendengué, L., Blangy, A., Zeghouf, M., et al. (2017). Allosteric inhibition of the guanine nucleotide exchange factor DOCK5 by a small molecule. Sci. Rep. 7, 14409. doi:10.1038/s41598-01713619-2

Frances, D., Sharma, N., Pofahl, R., Maneck, M., Behrendt, K., Reuter, K., et al. (2015). A role for Racl activity in malignant progression of sebaceous skin tumors. Oncogene 34, 5505-5512. doi:10.1038/onc.2014.471

Gao, Y., Dickerson, J. B., Guo, F., Zheng, J., and Zheng, Y. (2004). Rational design and characterization of a Rac GTPase-specific small molecule inhibitor. Proc. Natl. Acad. Sci. U.S.A. 101, 7618-7623. doi:10.1073/pnas.0307512101

Gao, Y., Xing, J., Streuli, M., Leto, T., and Zheng, Y. (2002). Trp(56) of rac1 specifies interaction with a subset of guanine nucleotide exchange factors. J. Biol. Chem. 276, 47530-47541. doi:10.1074/jbc.M108865200

Gatenby, R. A. (2009). A change of strategy in the war on cancer. Nature 459, 508-509. doi:10.1038/459508a

Gillan, M. J., Alfè, D., and Michaelides, A. (2016). Perspective: how good is DFT for water?. J. Chem. Phys. 144, 130901. doi:10.1063/1.4944633

Gohlke, H., Kiel, C., and Case, D. A. (2003). Insights into protein-protein binding by binding free energy calculation and free energy decomposition for the RasRaf and Ras-RalGDS complexes. J. Mol. Biol. 330, 891-913. doi:10.1016/s00222836(03)00610-7

Habets, G. G., Scholtes, E. H., Zuydgeest, D., Van Der Kammen, R. A., Stam, J. C., Berns, A., et al. (1994). Identification of an invasion-inducing gene, Tiam-1, that encodes a protein with homology to GDP-GTP exchangers for Rho-like proteins. Cell 77, 537-549. doi:10.1016/0092-8674(94)90216-x

Huo, S., Massova, I., and Kollman, P. A. (2001). Computational alanine scanning of the 1:1 human growth hormone-receptor complex. J. Comput. Chem. 23, 15-27. doi:10.1002/jcc. 1153

Jakalian, A., Jack, D. B., and Bayly, C. I. (2002). Fast, efficient generation of highquality atomic charges. AM1-BCC model: II. Parameterization and validation. J. Comput. Chem. 23, 1623-1641. doi:10.1002/jcc.10128

Jakalian, A., Bush, B. L., Jack, D. B., and Bayly, C. I. (2000). Fast, efficient generation of high-quality atomic charges. AM1-BCC model: I. Method. J. Comput. Chem. 21, 132-146. doi:10.1002/(SICI)1096-987X(20000130)21:2<132::AID-JCC5>3. $0 . \mathrm{CO} ; 2-\mathrm{P}$

Junmei, W., and Peter, A. K. (2010). Automatic parameterization of force field by systematic search and genetic algorithms. J. Comput. Chem. 22, 1219-1228. doi:10.1002/jcc.1079

Kamai, T., Shirataki, H., Nakanishi, K., Furuya, N., Kambara, T., Abe, H., et al. (2010). Increased Racl activity and Pakl overexpression are associated with lymphovascular invasion and lymph node metastasis of upper urinary tract cancer. BMC Cancer 10, 164. doi:10.1186/1471-2407-10-164
Kuhn, B., and Kollman, P. A. (2000). A ligand that is predicted to bind better to avidin than biotin: insights from computational fluorine scanning. J. Am. Chem. Soc. 122, 3909-3916. doi:10.1021/ja994180s

Larkin, M. A., Blackshields, G., Brown, N. P., Chenna, R., Mcgettigan, P. A., Mcwilliam, H., et al. (2007). Clustal W and clustal X version 2.0. Bioinformatics 23, 2947-2948. doi:10.1093/bioinformatics/btm404

Lee, S. C., Sim, N., Clement, M. V., Yadav, S. K., and Pervaiz, S. (2007). Dominant negative Rac1 attenuates paclitaxel-induced apoptosis in human melanoma cells through upregulation of heat shock protein 27: a functional proteomic analysis. Proteomics 7, 4112-4122. doi:10.1002/pmic.200700386

Levay, M., Krobert, K. A., Wittig, K., Voigt, N., Bermudez, M., Wolber, G., et al. (2013). NSC23766, a widely used inhibitor of Rac1 activation, additionally acts as a competitive antagonist at muscarinic acetylcholine receptors. J. Pharmacol. Exp. Therapeut. 347, 69-79. doi:10.1124/jpet.113.207266

Maier, J. A., Martinez, C., Kasavajhala, K., Wickstrom, L., Hauser, K. E., and Simmerling, C. (2015). ff14SB: improving the accuracy of protein side chain and backbone parameters from ff99SB. J. Chem. Theor. Comput. 11, 3696-3713. doi:10.1021/acs.jctc.5b00255

Malliri, A., Van Der Kammen, R. A., Clark, K., Van Der Valk, M., Michiels, F., and Collard, J. G. (2002). Mice deficient in the Rac activator Tiam1 are resistant to Ras-induced skin tumours. Nature 417, 867-871. doi:10.1038/nature00848

Martí-Renom, M. A., Stuart, A. C., Fiser, A., Sánchez, R., Melo, F., and Sali, A. (2000). Comparative protein structure modeling of genes and genomes. Annu. Rev. Biophys. Biomol. Struct. 29, 291-325. doi:10.1146/annurev.biophys.29. 1.291

Moreira, I. S., Fernandes, P. A., and Ramos, M. J. (2007). Computational alanine scanning mutagenesis--an improved methodological approach. J. Comput. Chem. 28, 644-654. doi:10.1002/jcc.20566

Morris, G. M., Huey, R., Lindstrom, W., Sanner, M. F., Belew, R. K., Goodsell, D. S., et al. (2009). AutoDock4 and AutoDockTools4: automated docking with selective receptor flexibility. J. Comput. Chem. 30, 2785-2791. doi:10.1002/ jcc. 21256

Myant, K. B., Cammareri, P., Mcghee, E. J., Ridgway, R. A., Huels, D. J., Cordero, J. B., et al. (2013). ROS production and NF- $\mathrm{BB}$ activation triggered by RAC1 facilitate WNT-driven intestinal stem cell proliferation and colorectal cancer initiation. Cell Stem Cell 12, 761-773. doi:10.1016/j.stem.2013.04.006

Pearson, W. R., and Lipman, D. J. (1988). Improved tools for biological sequence comparison. Proc. Natl. Acad. Sci. U.S.A. 85, 2444-2448. doi:10.1073/pnas.85.8. 2444

Remmert, M., Biegert, A., Hauser, A., and Söding, J. (2011). HHblits: lightning-fast iterative protein sequence searching by HMM-HMM alignment. Nat. Methods 9 (2), 173-175. doi:10.1038/nmeth.1818

Ruihua, H., Mengyi, Z., Chong, Z., Meng, Q., Xin, M., Qiulin, T., et al. (2016). RhoA regulates resistance to irinotecan by regulating membrane transporter and apoptosis signaling in colorectal cancer. Oncotarget 7, 87136-87146. doi:10. 18632/oncotarget. 13548

Ryckaert, J. P., Ciccotti, G., and Berendsen, H. (1977). Numerical integration of the cartesian equations of motion of a system with constraints: molecular dynamics of n-alkanes. J. Comput. Phys. 23, 327-341.

Shen, D. W., Su, A., Liang, X. J., Pai-Panandiker, A., and Gottesman, M. M. (2004). Reduced expression of small GTPases and hypermethylation of the folate binding protein gene in cisplatin-resistant cells. Br. J. Cancer 91, 270-276. doi:10.1038/sj.bjc.6601956

Sievers, F., Wilm, A., Dineen, D., Gibson, T. J., Karplus, K., Li, W., et al. (2011). Fast, scalable generation of high-quality protein multiple sequence alignments using Clustal Omega. Mol. Syst. Biol. 7, 539. doi:10.1038/msb.2011.75

Trott, O., and Olson, A. J. (2010). AutoDock Vina: improving the speed and accuracy of docking with a new scoring function, efficient optimization, and multithreading. J. Comput. Chem. 31, 455-461. doi:10.1002/jcc.21334

Um, K., Niu, S., Duman, J. G., Cheng, J. X., Tu, Y. K., Schwechter, B., et al. (2014). Dynamic control of excitatory synapse development by a Rac1 GEF/GAP regulatory complex. Dev. Cell 29, 701-715. doi:10.1016/j.devcel.2014.05.011

Valdés-Tresanco, M., Valdés-Tresanco, M., Valiente, P., and Moreno Frias, E. (2020). AMDock: a versatile graphical tool for assisting molecular docking with Autodock Vina and Autodock4. Biol. Direct 15 (1), 12. doi:10.1186/s13062-02000267-2 
Wang, C., Yan, G., Zhang, Y., Jia, X., and Bu, P. (2015). Long non-coding RNA MEG3 suppresses migration and invasion of thyroid carcinoma by targeting of Rac1. Neoplasma 62, 541-549. doi:10.4149/ neo_2015_065

Wang, J., Wolf, R., Caldwell, J., Kollman, P., and Case, D. (2004). Development and testing of a general amber force field. J. Comput. Chem. 25, 1157-1174. doi:10. $1002 /$ jcc. 20035

Worthylake, D. K., Rossman, K. L., and Sondek, J. (2000). Crystal structure of Racl in complex with the guanine nucleotide exchange region of Tiam1. Nature 408, 682. doi:10.1038/35047014

Worthylake, D., Rossman, K., and Sondek, J. (2000). Crystal structure of Rac1 in complex with the guanine nucleotide exchange region of Tiam1. Nature 408, 682. doi:10.1038/35047014

$\mathrm{Wu}, \mathrm{X}$., and Brooks, B. R. (2003). Self-guided Langevin dynamics simulation method. Chem. Phys. Lett. 381, 512-518. doi:10.1016/j. cplett.2003.10.013

Zeng, R. J., Zheng, C. W., Gu, J. E., Zhang, H. X., Xie, L., Xu, L. Y., et al. (2019). RACl inhibition reverses cisplatin resistance in esophageal squamous cell carcinoma and induces downregulation of glycolytic enzymes. Mol. Oncol. 13, 2010-2030. doi:10.1002/1878-0261.12548

Zhou, Y., Wang, Y., Zhou, W., Chen, T., Wu, Q., Chutturghoon, V. K., et al. (2019). YAP promotes multi-drug resistance and inhibits autophagy-related cell death in hepatocellular carcinoma via the RAC1-ROS-mTOR pathway. Cancer Cell Int. 19, 179. doi:10.1186/s12935-019-0898-7

Conflict of Interest: The authors declare that the research was conducted in the absence of any commercial or financial relationships that could be construed as a potential conflict of interest.

Copyright (๑) 2021 Zheng, Wu, Zeng, Lin, Xu, Li and Dong. This is an open-access article distributed under the terms of the Creative Commons Attribution License (CC $B Y$ ). The use, distribution or reproduction in other forums is permitted, provided the original author(s) and the copyright owner(s) are credited and that the original publication in this journal is cited, in accordance with accepted academic practice. No use, distribution or reproduction is permitted which does not comply with these terms. 